\title{
Magnetic monopole and confinement/deconfinement phase transition in SU(3) Yang-Mills theory
}

\author{
Akihiro Shibata* \\ Computing Research Center, High Energy Accelerator Research Organization (KEK) \\ and Graduate University for Advanced Studies (Sokendai), Tsukuba 305-0801, Japan \\ E-mail: akihiro.shibata@kek.jp
}

\section{Kei-Ichi Kondo}

Department of Physics, Graduate School of Science, Chiba University, Chiba 263-8522, Japan

E-mail: kondok@faculty.chiba-u.jp

\section{Seikou Kato}

Fukui National College of Technology, Sabae, Fukui 916-8507, Japan

E-mail: skato@fukui-nct.ac.jp

\section{Toru Shinohara}

Department of Physics, Graduate School of Science, Chiba University, Chiba 263-8522, Japan

E-mail: sinoharaegraduate.chiba-u.jp

\begin{abstract}
We have proposed the non-Abelian dual superconductivity in SU(3) Yang-Mills theory for the mechanism of quark confinement, and we presented the numerical evidences in preceding lattice conferences by using the proposed gauge link decomposition to extract magnetic monopole in the gauge invariant way. In this talk, we focus on the dual Meissner effects in view of the magnetic monopole in SU(3) Yang-Mills theory. We measure the chromoelectric and chromomagnetic flux due to a pair of quark and antiquark source at finite temperature. Then, we measure the correlation function of Polyakov loops and Polyakov loop average at various temperatures, and investigate chromomagnetic monopole current induced by chromo-magnetic flux in both confinement and deconfinement phase. We will discuss the role of the chromoelectric monopole in confinement/deconfinement phase transition.
\end{abstract}

The 32nd International Symposium on Lattice Field Theory,

23-28 June, 2014

Columbia University New York, $N Y$

${ }^{*}$ Speaker. 


\section{Introduction}

The dual superconductivity is a promising mechanism for quark confinement [1]. To establish the dual superconductivity picture, we must show the magnetic monopoles play the dominant role in quark confinement. We have presented a new formulation Yang-Mills (YM) theory and proposeed the non-Abelain dual superconductivity picture for $S U$ (3) Yang-Mills theory (for a review see [2]). We have presented a lattice version of a new formulation of $S U(N)$ YM theory[3], that gives the decomposition of a gauge link variable $U_{x, \mu}=X_{x, \mu} V_{x, \mu}$, which is suited for extracting the dominant mode, $V_{x, \mu}$, for quark confinement in the gauge independent way. In the case of the $S U(2)$ YM theory, the decomposition of the gauge link variable is given by a compact representation of the Cho-Duan-Ge-Faddeev-Niemi (CDGFN) decomposition [4] on a lattice [5][6][7]. For the $S U(N)$ YM theory, the new formula for the decomposition of a gauge link variable is constructed as an extension of the $S U(2)$ case. Our formulation can overcome the problems in the Abelian projection method: the magnetic monopole dominant is obtained only in special gauges such as the maximal Abelian (MA) gauge and the Laplacian Abelian gauge, and the Abelian projection itself breaks the gauge symmetry as well as color symmetry (global symmetry).

To the SU(3) YM theory, we have applied the minimal option. The minimal option is obtained for the stability group of $\tilde{H}=U(2) \cong S U(2) \times U(1)$, which is suitable for the Wilson loop in the fundamental representation. This fact is derived from the non-Abelian Stokes theorem [21]. Then, we have demonstrated the gauge-independent (invariant) restricted $V$-field dominance (or conventionally called Abelian dominance) and the gauge independent non-Abelian magnetic monopole dominance [12][10][[13][14][20]. The dual Meissner effect in YM theory must be examined by measuring the distribution of the chromoelectric field strength (or chromo flux) as well as the magnetic monopole current created by a static quark-antiquark pair. In the $S U(2)$ case, the extracted field corresponding to the stability group $\tilde{H}=U(1)$ shows the dual Meissner effect [8], which is a gauge invariant version of the Abelian projection in MA gauge. In the $S U(3)$ case, there are many works on chromo flux by using Wilson line/loop operator, e.g., [23][24][25]. At the previous conference, we have demonstrated the non-Abelian dual Meissner effect[15]. By applying our new formulation to the $S U(3)$ YM theory, we have given the numerical evidence of the non-Abelian dual Meissner effect claimed by us, and found the chromoelectric flux tube by measuring the chromo flux created by a static quark-antiquark pair. We have determined that the type of vacuum for $S U(3)$ YM theory is of type I, which is in sharp contrast to the $S U$ (2) case: the border of type I and type II [17] or of week type I [8].

In this talk, we focus on the confinement/deconfinement phase transition and the non-Abelian dual superconductivity at finite temperature: We measure a Polyakov loop average and correlation functions of the Polyakov loops which are defined for both the original YM field and extracted $V$-field to examine the $V$-field dominance in the Polyakov loop at finite temperature. Then, we measure the chromoelectric flux between a pair of static quark and antiquark of the Polyakov loops, and investigate its relevance to the phase transition and the non-Abelian dual Meissner effect.

\section{Method}

We introduce a new formulation of the lattice YM theory in the minimal option, which extracts the dominant mode of the quark confinement for $S U(3)$ YM theory[20, 14], since we consider the quark confinement in the fundamental representation. Let $U_{x, \mu}=X_{x, \mu} V_{x, \mu}$ be a decomposition of the YM link variable $U_{x, \mu}$, where $V_{x, \mu}$ could be the dominant mode for quark confinement, and $X_{x, \mu}$ the remainder part. The YM field and the decomposed new variables are transformed by full $S U(3)$ gauge transformation $\Omega_{x}$ such that $V_{x, \mu}$ is 
transformed as the gauge link variable and $X_{x, \mu}$ as the site variable:

$$
\begin{aligned}
& U_{x, \mu} \longrightarrow U_{x, v}^{\prime}=\Omega_{x} U_{x, \mu} \Omega_{x+\mu}^{\dagger}, \\
& V_{x, \mu} \longrightarrow V_{x, v}^{\prime}=\Omega_{x} V_{x, \mu} \Omega_{x+\mu}^{\dagger}, X_{x, \mu} \longrightarrow X_{x, v}^{\prime}=\Omega_{x} X_{x, \mu} \Omega_{x}^{\dagger} .
\end{aligned}
$$

The decomposition is given by solving the defining equation:

$$
\begin{aligned}
& D_{\mu}^{\varepsilon}[V] \mathbf{h}_{x}:=\frac{1}{\varepsilon}\left[V_{x, \mu} \mathbf{h}_{x+\mu}-\mathbf{h}_{x} V_{x, \mu}\right]=0, \\
& g_{x}:=e^{i 2 \pi q / 3} \exp \left(-i a_{x}^{0} \mathbf{h}_{x}-i \sum_{j=1}^{3} a_{x}^{(j)} \mathbf{u}_{x}^{(j)}\right)=1,
\end{aligned}
$$

where $\mathbf{h}_{x}$ is an introduced color field $\mathbf{h}_{x}=\xi\left(\lambda^{8} / 2\right) \xi^{\dagger} \in[S U(3) / U(2)]$ with $\lambda^{8}$ being the Gell-Mann matrix and $\xi$ an $S U$ (3) group element. The variable $g_{x}$ is an undetermined parameter from Eq.(2.2a), $\mathbf{u}_{x}^{(j)}$,s are $s u(2)$-Lie algebra valued, and has $q_{x}$ an integer value $0,1,2$. These defining equations can be solved exactly [19], and the solution is given by

$$
\begin{array}{ll}
X_{x, \mu}=\widehat{L}_{x, \mu}^{\dagger} \operatorname{det}\left(\widehat{L}_{x, \mu}\right)^{1 / 3} g_{x}^{-1}, & V_{x, \mu}=X_{x, \mu}^{\dagger} U_{x, \mu}=g_{x} \widehat{L}_{x, \mu} U_{x, \mu}, \\
\widehat{L}_{x, \mu}=\left(L_{x, \mu} L_{x, \mu}^{\dagger}\right)^{-1 / 2} L_{x, \mu}, & L_{x, \mu}=\frac{5}{3} \mathbf{1}+\frac{2}{\sqrt{3}}\left(\mathbf{h}_{x}+U_{x, \mu} \mathbf{h}_{x+\mu} U_{x, \mu}^{\dagger}\right)+8 \mathbf{h}_{x} U_{x, \mu} \mathbf{h}_{x+\mu} U_{x, \mu}^{\dagger} .
\end{array}
$$

Note that the above defining equations correspond to the continuum version: $D_{\mu}[\mathscr{V}] \mathbf{h}(x)=0$ and $\operatorname{tr}\left(\mathbf{h}(x) \mathscr{X}_{\mu}(x)\right)$ $=0$, respectively. In the naive continuum limit, we have reproduced the decomposition $\mathbf{A}_{\mu}(x)=\mathbf{V}_{\mu}(x)+$ $\mathbf{X}_{\mu}(x)$ in the continuum theory [19] as

$$
\begin{aligned}
& \mathbf{V}_{\mu}(x)=\mathbf{A}_{\mu}(x)-\frac{4}{3}\left[\mathbf{h}(x),\left[\mathbf{h}(x), \mathbf{A}_{\mu}(x)\right]\right]-i g^{-1} \frac{4}{3}\left[\partial_{\mu} \mathbf{h}(x), \mathbf{h}(x)\right], \\
& \mathbf{X}_{\mu}(x)=\frac{4}{3}\left[\mathbf{h}(x),\left[\mathbf{h}(x), \mathbf{A}_{\mu}(x)\right]\right]+i g^{-1} \frac{4}{3}\left[\partial_{\mu} \mathbf{h}(x), \mathbf{h}(x)\right] .
\end{aligned}
$$

The decomposition is uniquely obtained as the solution (2.3) of Eqs.(2.2), if color fields $\left\{\mathbf{h}_{x}\right\}$ are obtained. To determine the configuration of color fields, we use the reduction condition to formulate the new theory written by new variables $\left(X_{x, \mu}, V_{x, \mu}\right)$ which is equipollent to the original YM theory. Here, we use the reduction functional:

$$
F_{\text {red }}\left[\mathbf{h}_{x}\right]=\sum_{x, \mu} \operatorname{tr}\left\{\left(D_{\mu}^{\varepsilon}\left[U_{x, \mu}\right] \mathbf{h}_{x}\right)^{\dagger}\left(D_{\mu}^{\varepsilon}\left[U_{x, \mu}\right] \mathbf{h}_{x}\right)\right\},
$$

and then color fields $\left\{\mathbf{h}_{x}\right\}$ are obtained by minimizing the functional (2.5).

\section{Lattice result}

We generate YM gauge configurations $\left\{U_{x, \mu}\right\}$ at finite temperature using the standard Wilson action. We set up a lattice $L^{3} \times N_{T}\left(L=24, N_{T}=6\right)$ and the control the temperature by changing the parameter $\beta$ : $\beta=5.8,5.9,6.0,6.1,6.2,6.3$. We generate 500 configurations for each $\beta$. In the measurement of the Polyakov loop and Wilson loop, we apply the APE smearing technique to reduce noises [29]. The gauge link decomposition $U_{x, \mu}=X_{x, \mu} V_{x, \mu}$ is obtained by the formula (2.3) given in the previous section, after the color field configuration $\left\{\mathbf{h}_{x}\right\}$ is obtained by solving the reduction condition of minimizing the functional eq(2.5) for each gauge configuration $\left\{U_{x, \mu}\right\}$.

Figure 1 shows the distribution of space-averaged Polyakov loops for each configuration:

$$
P_{U}:=L^{-3} \sum_{\{\vec{x}\}} \operatorname{tr}\left(\prod_{t=1}^{N_{T}} U_{(\vec{x}, t), 4}\right), \quad P_{V}:=L^{-3} \sum_{\{\vec{x}\}} \operatorname{tr}\left(\prod_{t=1}^{N_{T}} V_{(\vec{x}, t), 4}\right) .
$$



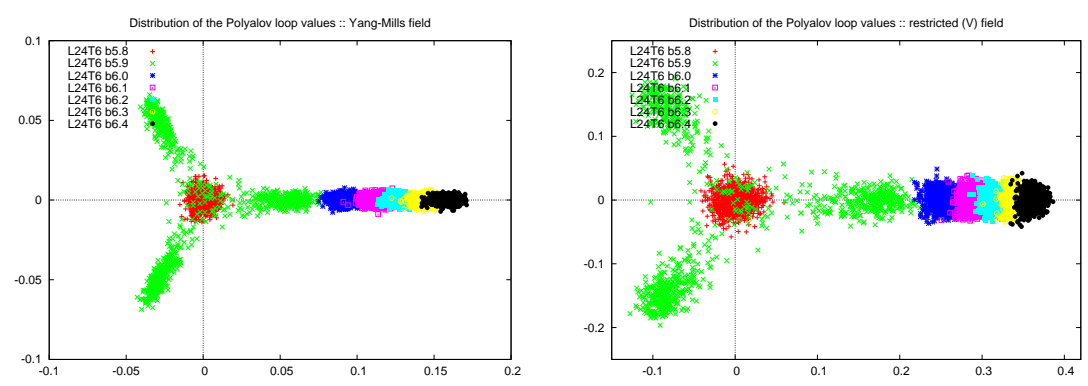

Figure 1: The distribution of the space-averaged Polyakov loop for each configuration:(Left) For the YM field. (Right) For the restricted field.

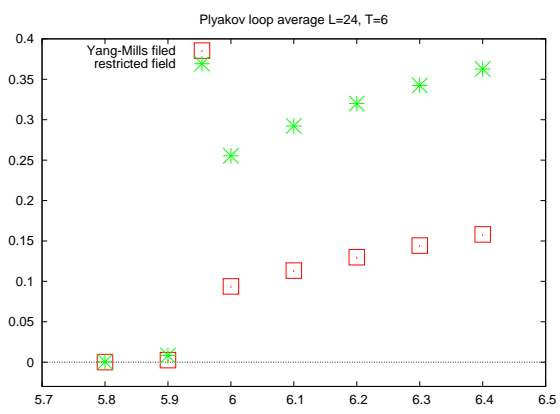

Figure 2: Parameter $\beta$ dependence of the Polyakov loop average: Red plots show $\left\langle P_{U}\right\rangle$ v.s $\beta$, green ones $\left\langle P_{V}\right\rangle$ v.s $\beta$.
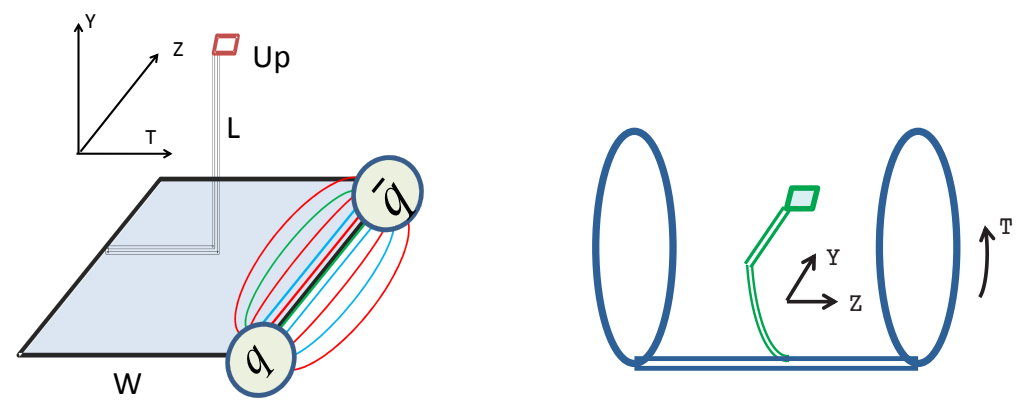

Figure 3: (Left) The connected operator $W L U_{p} L^{\dagger}$ between a plaquette $U_{p}$ and the Wilson loop $W$. (Right) Measurement of the chromo-flux at finite temperature via Polyakov loop.

The left panel Fig 1 shows the distribution of $P_{U}$ for the YM field for each configuration, and the right panel shows the distribution of $P_{V}$ for the restricted field ( $V$-field) for the relevant configurations. Then, we obtain the Polyakov loop average, which is the conventional order parameter for confinement and deconfinement phase transition in SU (3) YM theory. Figure 2 shows combined polts of the Polyakov loop average for the YM field $\left\langle P_{U}\right\rangle$ and the restricted field $\left\langle P_{V}\right\rangle$. Each plot shows the same critical temperature of confinement/deconfinement phase transition. These show the extracted $V$-field reproduces the phase transition at finite temperature. We can also show the restricted field ( $V$-field) dominance in the Polyakov loop correlation functions, which was presented at the last conference[18].

Next, we investigate the non-Abelian dual Meissner effect at finite temperature. Note that at finite tem- 

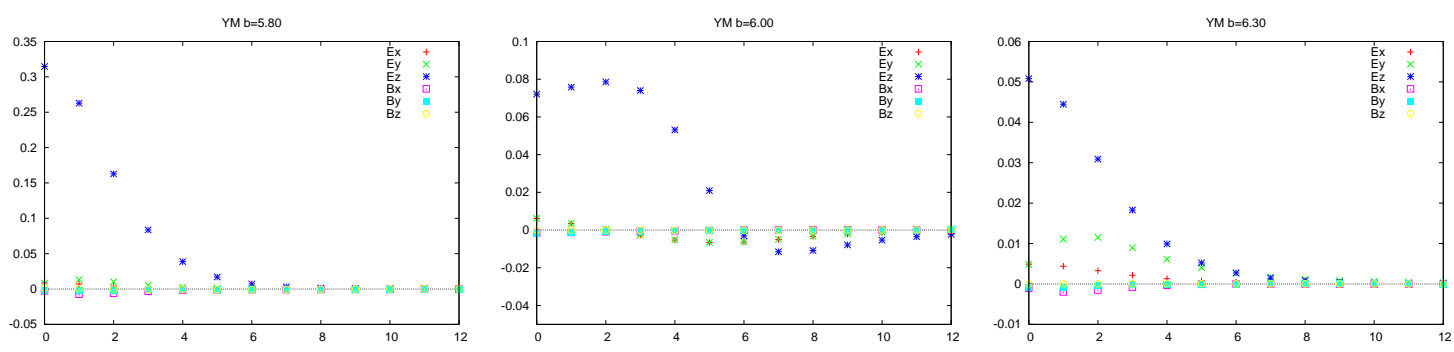

Figure 4: chromo-flux created by a pair of Polyakov loops of the YM field. (left) $\beta=5.80$, (middle ) $\beta=6.00$, (right) $\beta=6.30$
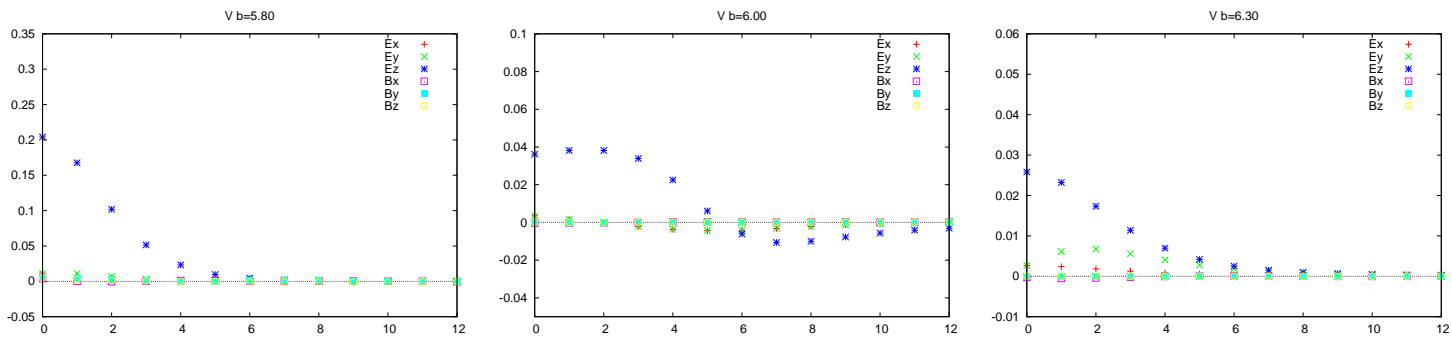

Figure 5: chromo-flux created by a pair of Polyakov loops of the restricted field. (left) $\beta=5.80$, (middle ) $\beta=6.00$, (right) $\beta=6.30$

perature we must use the operator with the same size in the temporal direction, and the quark and antiquark pair is replaced by a pair of the Polyakov loop with the opposite direction. To investigate the chromo flux, we use the gauge invariant correlation function which is used at zero temperature. The chromo flux created by a quark-antiquark pair is measured by using a gauge-invariant connected correlator of the Wilson loop [26]:

$$
\rho_{W}:=\frac{\left\langle\operatorname{tr}\left(U_{p} L^{\dagger} W L\right)\right\rangle}{\langle\operatorname{tr}(W)\rangle}-\frac{1}{3} \frac{\left\langle\operatorname{tr}\left(U_{p}\right) \operatorname{tr}(W)\right\rangle}{\langle\operatorname{tr}(W)\rangle},
$$

where $W$ represents the source of a quark-antiquark pair settled by the Wilson loop in Z-T plane, $U_{p}$ a plaquette variable as the probe operator for measuring the field strength, and $L$ the Wilson line connecting the source $W$ and the probe $U_{p}$. (see the left panel of Figure 3). The symbol $\langle\mathscr{O}\rangle$ denotes the average of the operator $\mathscr{O}$ over the space and the ensemble of the configurations. Note that this is sensitive to the field strength rather than the disconnected one. Indeed, in the naive continuum limit, the connected correlator $\rho_{W}$ is given by $\rho_{W} \stackrel{\varepsilon \rightarrow 0}{\simeq} g \varepsilon^{2}\langle\mathscr{F} \mu v\rangle_{q \bar{q}}:=\frac{\left\langle\operatorname{tr}\left(g \varepsilon^{2} \mathscr{F}_{\mu v} L^{\dagger} W L\right)\right\rangle}{\langle\operatorname{tr}(W)\rangle}+O\left(\varepsilon^{4}\right)$. Thus, the chromo field strength is given by $F_{\mu \nu}=\sqrt{\frac{\beta}{6}} \rho_{W}$.

Figure 4 and 5 show the measurement of chromo flux for Yang-Mills field and restricted field $(V$-field) at finite temperature. The chromo-flux of quark-antiquark pair is measured on the plane at $z=1 / 3 R$ for a given quark at $z=0$ and an antiquark at $z=R$ by moving the probe, $U_{p}$ or $V_{p}$ along the y-direction. We find the restricted field dominance for the chromo-flux tube at finite temperature as well as zero temperature

At low temperature (see left panels of Fig.4 and Fig.5), we observe the chromoelectric flux tube such that only the $E_{z}$ component in the direction connecting a quark and antiquark pair is observed, while the other components take vanishing values. This is consistent with the result Ref.[30], though they use the different operator for the flux measurements.

At high temperature $\left(T>T_{C}\right.$ ) (see right panels of Fig. 4 and Fig.5), we can observe no more squeezing of the chromoelectric flux tube, and non-vanishing $E_{y}$ component in the chromoelectric flux. This shows the 
disappearance of the dual Meissner effect at high temperature.

Then, we investigate the magnetic (monopole) current due to the magnetic condensation:

$$
k_{\mu}(x)=\frac{1}{2} \varepsilon_{\mu v \alpha \beta}\left(F[V]_{\alpha \beta}(x+\hat{v})-F[V]_{\alpha \beta}(x)\right)
$$

Note that the magnetic monopole current eq(3.3) must have vanishing value if there exists no magnetic monopole condensation, since the right-hand side of eq(3.3) is the Bianchi identity. Therefore, the magnetic monopole current can be the order parameter of dual superconductivity. Figure 6 shows the measurements for the magnetic current eq(3.3). We observe the appearance and disappearance of the magnetic monopole current at low and high temperature, respectively.

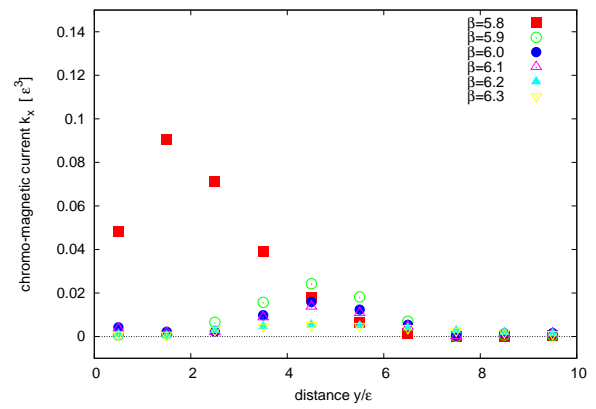

Figure 6: The magnetic carrent (monopole) induced by a pair of Polyakov loops.

\section{Summary and outlook}

We have investigated the non-Abelian dual Meissner effect at finite temperature by measuring the chromo flux due to a pair of quark and antiquark source represented by a pair of the Polyakov loops. Using our proposal for a new formulation of Yang-Mills theory on a lattice, we ware able to extract the dominant mode for quark confinement as the restricted field ( $V$-field), and confirmed that the restricted field dominance at finite temperature. We have observed no more squeezing of the chromoelectric flux tube due to the dual Meissner effect. We have also measured the magnetic (monopole) current in both the confinement and deconfinement phase, and observed that the confinement/deconfinement phase transition is associated to appearance and disappearance of magnetic (monopole) current. This is the evidence that the confinement/deconfinement phase transition is caused by appearance/disappearance of the non-Abelian dual superconductivity.

\section{Acknowledgement}

This work is supported by Grant-in-Aid for Scientific Research (C) 24540252 from Japan Society for the Promotion Science (JSPS), and also in part by JSPS Grant-in-Aid for Scientific Research (S) 22224003. The numerical calculations are supported by the Large Scale Simulation Program No.12-13 (FY2012), No. 12/13-20 (FY2012/13) and No.13/14-23 (FY2013-2014) of High Energy Accelerator Research Organization (KEK).

\section{References}

[1] Y. Nambu, Phys. Rev. D10, 4262(1974); G. 't Hooft, in High Energy Physics, edited by A. Zichichi (Editorice Compositori, Bologna, 1975); S. Mandelstam, Phys. Report 23, 245(1976); A.M. Polyakov, Nucl. Phys. B120, 429(1977). 
[2] K.-I. Kondo, S. Kato, A. Shibata, T. Shinohara, CHIBA-EP-209, KEK-PREPRINT-2014-23, e-Print: arXiv:1409.1599 [hep-th]

[3] K.-I. Kondo, T. Murakami and T. Shinohara, Eur. Phys. J. C 42, 475 (2005); K.-I. Kondo, T. Murakami and T. Shinohara, Prog. Theor. Phys. 115, 201 (2006).; K.-I. Kondo, T. Shinohara and T. Murakami, Prog.Theor. Phys. 120, 1 (2008)

[4] Y.M. Cho, Phys. Rev. D 21, 1080 (1980). Phys. Rev. D 23, 2415 (1981); Y.S. Duan and M.L. Ge, Sinica Sci., 11, 1072(1979); L. Faddeev and A.J. Niemi, Phys. Rev. Lett. 82, 1624 (1999); S.V. Shabanov, Phys. Lett. B 458, 322 (1999). Phys. Lett. B 463, 263 (1999).

[5] S. Ito, S. Kato, K.-I. Kondo, A. Shibata and T. Shinohara, Phys.Lett. B645 67-74 (2007)

[6] A. Shibata, S. Kato, K.-I. Kondo, T. Murakami, T. Shinohara and S. Ito, Phys.Lett. B653 101-108 (2007)

[7] S. Kato, K.-I. Kondo, A. Shibata and T. Shinohara, PoS(LAT2009) 228.

[8] S. Kato, K.-I. Kondo and A. Shibata, e-Print: arXiv:1407.2808 [hep-lat], to be published in Phys. Rev. D

[9] A. Shibata, S. Kato, K.-I. Kondo, T. Shinohara and S. Ito, POS(LATTICE2007) 331, arXiv:0710.3221 [hep-lat]

[10] A. Shibata, S. Kato, K.-I. Kondo, T. Shinohara and S. Ito, PoS(LATTICE 2008) 268, arXiv:0810.0956 [hep-lat]

[11] Shinya Gongyo, Takumi Iritani and Hideo Suganuma, Phys.Rev. D86 (2012) 094018

[12] K.-I. Kondo, A.Shibata, T. Shinohara, T. Murakami, S. Kato and S. Ito, Phys. Lett. B669, 107 (2008)

[13] A. Shibata, K.-I. Kondo, S.Kato, S. Ito, T. Shinohara and N. Fukui, PoS LAT2009 (2009) 232, arXiv:0911.4533 [hep-lat].

[14] A. Shibata, K.-I. Kondo, S. Kato and T. Shinohara, PoS(Lattice 2010) 286

[15] A. Shibata, K.-I. Kondo, S. Kato and T. Shinohara, PoS LATTICE2012 (2012) 215

[16] A. Shibata, K.-I. Kondo, S. Kato and T. Shinohara, PoS ConfinementX (2012) 052, : arXiv:1302.6865 [hep-lat]

[17] A. Shibata, K.-I. Kondo, S. Kato and T. Shinohara, Phys.Rev. D87 (2013) 5, 054011, arXiv:1212.6512[hep-lat]

[18] Akihiro Shibata, Kei-Ichi Kondo, Seikou Kato, Toru Shinohara, PoS LATTICE2013 (2014)

[19] A. Shibata, K.-I. Kondo and T. Shinohara, Phys.Lett.B691:91-98 (2010), arXiv:0911.5294[hep-lat]

[20] K.-I. Kondo, A. Shibata, T. Shinohara, S. Kato, Phys.Rev. D83 (2011) 114016, arXiv:1007.2696 [hep-th]

[21] K.-I. Kondo, Phys.Rev.D77, 085029 (2008)

[22] K.-I. Kondo and A. Shibata, CHIBA-EP-170, KEK-PREPRINT-2007-73, arXiv:0801.4203[hep-th]

[23] M. S. Cardaci, P. Cea, L. Cosmai, R. Falcone and A. Papa, Phys.Rev.D83, 014502 (2011)

[24] N. Cardoso, M. Cardoso, P. Bicudo, Phys.Rev. D84 (2011) 054508 , arXiv:1107.1355 [hep-lat]

[25] Cea, Cosmai and Papa, Phys. Rev. D86, 054501 (2012)

[26] A. Di Giacomo, M. Maggiore, and S. Olejnik, Phys. Lett. B236, 199 (1990); Nucl. Phys. B347, 441 (1990).

[27] Y. Matsubara, S. Ejiri and T. Suzuki, NPB Poc. suppl 34, 176 (1994)

[28] R.G.Edwards, U.M. Heller and T.R.Klassen, Phys. Rev. Lett. 80, 3448-3451 (1998).

[29] M. Albanese et al. (APE Collaboration), Phys. Lett. B192,163 (1987).

[30] P. Cea, L. Cosmai, F. Cuteri, and A.Papa, Phys. Rev. D89, 094505 (2014), arXiv:1404.1172 [hep-lat] 